\title{
Analysis and Configuration of Boundary Difference Calculations
}

\author{
Simon Dacey, Lei Song, Lei Zhu, and Shaoning Pang \\ Unitec Institute of Technology, \\ Carrington Road, Mt. Albert, Auckland, New Zealand
}

\begin{abstract}
In the field of land management, stakeholders (people) everywhere have many disputes over the location of boundaries between private land and public land. We find that the stakeholders disagree with each other over boundaries. We propose an approach that helps people to come to an agreement on position of boundaries (including pixel-based approach, polygon-based approach and middle boundary approach). The experiments are carried out on data relating to public parks in Auckland, New Zealand. The results of the experiments highlight the differences between different stakeholder's percieved boundaries.
\end{abstract}

Keywords: Boundary Negotiation, Boundary Disputes, Polygon-based Boundary Calcluation, GPS-based Boundary Detection

\section{Introduction}

Land Management is the process of managing the use and development (in both urban and rural settings) of land resources. Land resources include organic agriculture, reforestation, water resource management and eco-tourism projects, is called public land. Private property is a legal designation of the ownership of property by non-governmental legal entities. The use of public land for private purposes, known as encroachment, has been identified as a problem affecting public parks in the Auckland region. Every boundary conflict contains a strong spatial component [1]. The spatial location of a public park is defined by its boundary. However there may be several different versions of the same boundary for a park. Perception of encroachment depends upon the viewpoint of the stakeholder. The stakeholders are residents living around the parks, non-residents who use the parks, organised sports groups and representatives of the Auckland City Council (including managers, councillors and surveyors). In land management, arguments occur over boundaries between stakeholders.

\section{Related Work}

This sections examines research work that has been carried out in related areas. The related areas are the mathematical formulas used to calculate the differences between two sets of data (boundary or area) and the magnitude of the 
differences. A search of the literature reveals that people use the Hidden Markov Model [2] [3],the Boundary Element Method [4] [5] and point-set-based [6] [7] [8] to detect differences in boundaries. Hidden Markov models (HMM) are studied for the purpose of planar shape classification using curvature coefficients. A discrete-time HMM is a probabilistic model that describes a random sequence as the indirect observation of an underlying (hidden) random sequence where this hidden process in Markovian. The boundary element method (BEM) is a numerical computational method of solving linear partial differential equations which have been formulated as integral equations. A point-set-based model is developed for areal objects from a perspective that incorporates spatial cognition. This model is called with point-set-based regions (PSBR). Computing spatial relationships between two PSBRs using the derived areal objects consists of looking at topological relationships, directional relationships, metric relationships, distance between centroids, average distance and Hausdorff distance.

\subsection{Motivation}

After studying the methods listed above, we have found that they give an accurate detection of the differences. However, these works does not really solve the arguments among stakeholders, due to these methods not providing a possible solution for the stakeholders. We analysed the pixel-based calculation and proposed a polygon-based calculation to form a new point of view for the stakeholders, upon which they can base negotiations to solve the boundary dispute.

\section{Methodology}

Land use conflict occurs whenever land-use stakeholders have incompatible interests related to land areas that result in negative effects [9]. In order to resolve the arguments over boundaries, we examine two existing approaches: a) Pixelbased Approach and b) Polygon-based Approach and we then build upon the two approaches above to propose a new approach: Middle-boundary Approach.

\subsection{Pixel-based approach}

Given a set of $n$ sequential GPS coordinate pairs $G=\left\{\left(L o_{1}, L a_{1}\right), \ldots,\left(L o_{n}, L a_{n}\right)\right\}$ for one area, we firstly transfer them into integer coordinates according to certain predefined precision, for example $C=\left\{\left(X_{1}, Y_{1}\right), \ldots,\left(X_{n}, Y_{n}\right) \mid X_{i}=\right.$ $\left.\operatorname{round}\left(L o_{i} \times 1000\right), Y_{i}=\operatorname{round}\left(L a_{i} \times 1000\right) \forall i \in\{1, \ldots, n\}\right\}$. Then we shift the coordinate origin somehow to fit the coordination set as,

$$
\begin{gathered}
C_{s}=\left\{\left(x_{1}, y_{1}\right), \ldots,\left(x_{n}, y_{n}\right) \mid x_{i}=X_{i}-\min (X)+1, y_{i}=Y_{i}-\min (Y)+1\right. \\
\forall i \in\{1, \ldots, n\}\}
\end{gathered}
$$

Now we have a bitmap with $n$ positive pixels. Next we sequentially connect each neighboring pair ( $n$-th point is the neighbor with $n$-1-th and 1 -st). For 
example, if have $\left(x_{i}, y_{i}\right)$ and $\left(x_{i+1}, y_{i+1}\right)$, we need to compute a set of pixels approximately connecting $\left(x_{i}, y_{i}\right)$ to $\left(x_{i+1}, y_{i+1}\right)$ and also as a edge of closed polygon. To achieve such closed approximate pixel edge, we simply approximate the $y$ coordination from a continuous series of $x$ connecting $x_{i}$ to $x_{i+1}$ and do the reversed, as follows (assume $x_{i} \leq x_{i+1}$ and $y_{i} \leq y_{i+1}$ )

$$
\begin{aligned}
& x=\left[x_{i}, x_{i}+1, \ldots, x_{i+1}\right] \\
& y=y_{i}+x \times \frac{y_{i+1}-y_{i}}{x_{i+1}-x_{i}} \\
& y=\operatorname{round}(y) \\
& y=\left[y_{i}, y_{i}+1, \ldots, y_{i+1}\right] \\
& x=x_{i}+y \times \frac{x_{i+1}-x_{i}}{y_{i+1}-y_{i}} \\
& x=\operatorname{round}(x) .
\end{aligned}
$$

The result with more points from (2) and (3) is taken as the edge point set between $\left(x_{i}, y_{i}\right)$ and $\left(x_{i+1}, y_{i+1}\right)$. Once all edges for one area if obtained, we can apply area fill algorithm to fill the edge graph and obtain a binary bitmap of that area. For area difference, we can simply do a matrix subtraction to compute following areas:

1. both $A_{1}$ and $A_{2}$ covers;

2. $A_{1}$ covers but $A_{2}$ not;

3. $A_{2}$ covers but $A_{1}$ not.

As any field area is represented by a binary matrix $M=\{0,1\}^{m \times n}$, where $m$ and $n$ denote the number of rows and columns respectively of the bitmap, thus the area is simply calculated by

$$
S=\operatorname{sum}(\operatorname{sum}(M)) \text {. }
$$

\subsection{Polygon-based Approach}

In the analytic geometry method, a boundary is seen as a polygon formed by connecting points sequentially. Given two boundaries $A=\left\{a_{1}, a_{2} \ldots a_{n}\right\}$ and $B=\left\{b_{1}, b_{2} \ldots b_{m}\right\}$, where the end points of $A$ and $B$ are clockwise distributed. There are four steps to find the difference $A-B$ (i.e. the area inside $A$ but outside $B$ ) and $B-A$ (i.e. the area inside $B$ but outside $A$ ).

1. Find all cross points $C$ between any edge pairs, one from $A$ and one from $B$. As each edge is a line segment, there are many existing algorithms for finding cross point between two given line segments. The result of this step is a set of points $C=c_{1}, c_{2}, \ldots c_{o}$, each cross point $c_{k}$ is associated with one edge $a_{i} a_{i+1}$ from $A$ and one edge $b_{j} b_{j+1}$ from $B$.

2. Form the difference polygons $D_{1}, D_{2}, \ldots, D_{o}$. As boundary $A$ have $o$ cross points with $B$, it is easy to imagine that there are $o$ difference polygons. And the $k$-th difference polygon $D_{k}$ is defined by cross point $c_{k}$, a sequence of points $A_{k}$ from $A$, cross point $c_{k+1}$ and a sequence of points $B_{k}$ from $B$. 
Note that when $k=o$ have $c_{k+1}=c_{1}$, as the 'next' for the last one in a circle is the first. Then, to determine the difference polygon $D_{k}$, we need to find out the sequences $A_{k}$ and $B_{k}$. To compute the sequences $A_{k}$, we need firstly check if $c_{k}$ and $c_{k+1}$ are associated with the same edge $a_{i} a_{i+1}$ in $A$. If so, means the boundary $B$ cross edge $a_{i} a_{i+1}$ at least twice and there is no end point from $A$ between $c_{k}$ and $c_{k+1}$, thus we have $A_{k}=\emptyset$. If not, say $c_{k}$ associates with $a_{i} a_{i+1}$ and $c_{k+1}$ associates with $a_{l} a_{l+1}$, then $A_{k}$ is the sequences $\left[a_{i+1}, a_{i+2}, \ldots, a_{l}\right]$. Also note that if have $i+1>l$, the sequences $A_{k}$ actually becomes $\left[a_{i+1}, a_{i+2}, \ldots, a_{n}, a_{1}, \ldots, a_{l}\right]$, always keep in mind that we are working on a 'circle'. Applying the same method, we can determine the sequences $B_{k}$ say $B_{k}=\left[b_{j+1}, b_{j+2}, \ldots, b_{p}\right]$. Recall that both $A$ and $B$ are clockwise distributed, so are the $A_{k}$ and $B_{k}$. Thus to define the difference polygon $D_{k}$, we need to use the inverse sequences $B_{k}^{\prime}=\left[b_{p}, \ldots, b_{j+2}, b_{j+1}\right]$ with is counterclockwised. By now we have the difference polygon $D_{k}=$ $c_{k} A_{k} c_{k+1} B_{k}^{\prime}$ computed. And so for all difference polygons $D_{1}, D_{2}, \ldots, D_{o}$

3. Determine which set of difference polygons belong to $A-B$ and which set to $B-A$. It is easy to conclude that for any neighboring difference polygon $D_{k}$ and $D_{k+1}$ should have the different identity, as if boundary of $A$ is 'outside' of $B$ between cross points $c_{k}$ and $c_{k+1}$ which indicates $D_{k}$ belongs to $A-B$, after the cross in $c_{k+1}$, the boundary of $A$ becomes 'inside' the boundary which indicates that $D_{k+1}$ belongs to $B-A$, and vice versa. Thus, we can simply determine the difference polygons belonging by finding out the identity of any difference polygon. This can be done by detect whether a point from $A$ (e.g. $a_{1}$ ) is inside polygon $B$. Make a ray from $a_{1}$ to any direction, if there is odd numbered cross point with polygon $B$, then $a_{1}$ is inside $B$; and if the cross number is even, then $a_{1}$ is outside $B$.

4. Compute the area of difference polygons. Given any polygon $A=a_{1} a_{2} \ldots a_{n}$ and its coordinate set $\left\{\left(x_{1}, y_{1}\right),\left(x_{2}, y_{2}\right), \ldots,\left(x_{n}, y_{n}\right)\right\}$, the area of $A$ can be computed as

$$
S_{A}=\frac{1}{2} \sum_{i=1}^{n}\left(x_{i} y_{i+1}-x_{i+1} y_{i}\right) .
$$

Note that when $i=n$, have $a_{n+1}=a_{1}$ and $x_{n+1}=x_{1}, y_{n+1}=y_{1}$.

\subsection{Middle-boundary Approach}

To find the middle-boundary, we propose a nearest neighbor based algorithm. Let $A=a_{1} a_{2} \ldots a_{n}$ be the boundary with more endpoints than $B=b_{1} b_{2} \ldots b_{m}$, we calculated a middle boundary $M=m_{1} m_{2} \ldots m_{n}$ have the same number of end points with $A$, each $m_{i}$ lays in the halfway of $a_{i}$ to its nearest neighbor in $\left\{b_{1}, b_{2}, \ldots, b_{m}\right\}$. To find such nearest neighbor, we need firstly compute a distance vector $D=R^{m \times 1}$ have

$$
D_{j}=\operatorname{dist}\left(a_{i}, b_{j}\right)=\sqrt{\left(x_{a i}-x_{b j}\right)^{2}+\left(y_{a i}-y_{b j}\right)^{2}} .
$$

Then find the $j$ with smallest $D_{j}$, and eventually compute the mean vector of $\left[x_{a i}, y_{a i}\right]$ and $\left[x_{b j}, y_{b j}\right]$. 


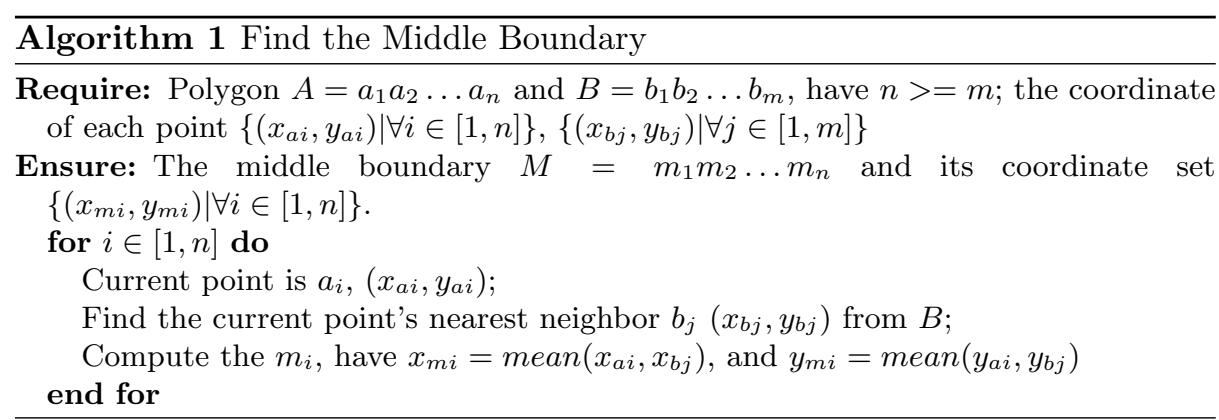

Once $M$ is determined, using algorithm mentioned in Section3.2, we can compute the difference area between $A$ to $M$ and $B$ to $M$.

\section{Experiments}

To demonstrate the boundary comparison techniques, for this paper, 20 parks from the Manukau and North Shore areas are selected as representative examples of the four categories of park discussed above.

\subsection{Experimental setup}

A number of experiments were conducted to study the effectiveness of the new approach. In this section we look at the data used in the experiments, the setup and procedure of the experiments and we discuss the results of the experiments.

\subsection{Location and Device}

GPS Data Collection carried out at 20 sites across North Shore and Manukau areas in Auckland. One GPS devices used: Leica Viva TPS. The data collected, using the Leica Viva TPS device, uses the Mt Eden 2000 co-ordinates system.

\subsection{Data}

The data used in the experiments is boundary data of New Zealand specifically the Auckland area.

1 GPS Boundary Data. The GPS boundary data has been collected during field visits to the selected parks.

2 Land Boundary Data. The Council boundary data is used to compare against the observed boundary data. 


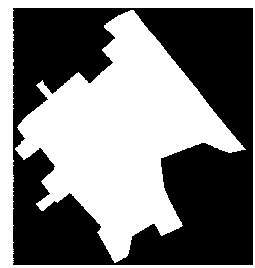

(a) Area covered by both sets of data

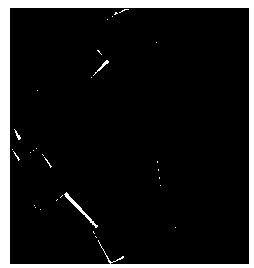

(b) Positive differences between data sets

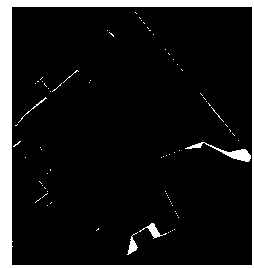

(c) Negative differences between data sets

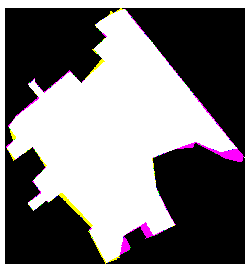

(d) Composite image of data

Fig. 1: Pixel Based Approach

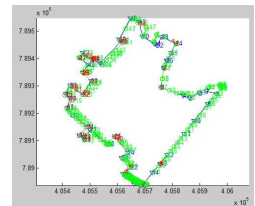

(a) Boundary cross points

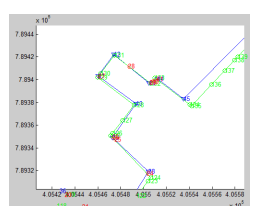

(b) Boundary cross points zoomed in view

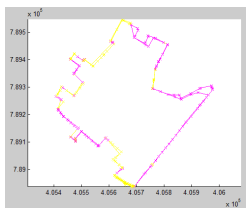

(c) Differences between polygons

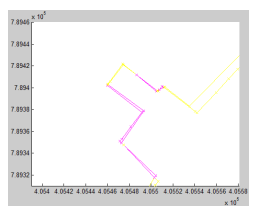

(d) Differences between polygons - zoomed in view

Fig. 2: Polygon Based Approach

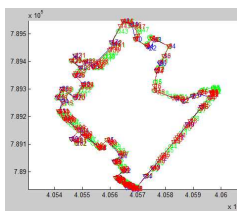

(a) Overview of(b) Zoomed-in Middle-Boundary view of MiddleApproach

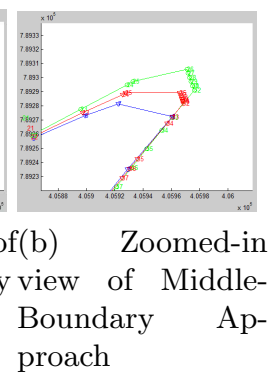

Fig. 3: Middle-Boundary Approach

\subsection{Discussion}

The argument from people for the analyzing frames illuminates the underlying causes of Park boundries in the geographic analysis of public land use. Geographic information systems (GIS) have long been applied in resolving municipal/local boundary conflicts (e.g. US political redistricting) [10]. This approach utilises both GIS and GPS in presenting and resolving boundary disputes. 
Table 1: Results of the polygon-based experiments

\begin{tabular}{ccccccc}
\hline & \multicolumn{3}{c}{ Polygon-based } & \multicolumn{3}{c}{ Middle-boundary } \\
Name & AreaOmG & AreaGmO & AreaOmM & AreaMmO & AreaGmM & AreaMmG \\
\hline Agincourt Reserve & 3050.970703 & 46.8359375 & 2566.961914 & 20.89550781 & 132.734375 & 590.8027344 \\
Aorere Park & 4635.09082 & 2241.937531 & 2224.094147 & 1173.392487 & 1138.012573 & 2480.46402 \\
Auburn Reserve & 12983.03125 & 0.544921875 & 7507.915039 & 111.6933594 & 0.13671875 & 5586.398438 \\
Beaumont Park & 351.6609802 & 854.4945984 & 291.2216797 & 363.8186035 & 596.5552368 & 166.3186951 \\
Dale Reserve & 0 & 0 & 0 & 0 & 13001.17609 & 3857.525452 \\
Diana Reserve & 234.1083984 & 179.5517578 & 118.3222656 & 93.94726563 & 88.72363281 & 118.9091797 \\
Gallaher Park & 1846.82312 & 2653.692627 & 1193.333954 & 1283.261169 & 1471.670105 & 754.7279663 \\
Holland Reserve & 2972.285156 & 319.7568359 & 1480.804688 & 9044.454102 & 502.2460938 & 10718.42871 \\
James Watson Park & 240.3355103 & 15420.63184 & 120.597168 & 6829.786682 & 9515.397919 & 1044.291077 \\
Jellicoe Park & 549.1130066 & 481.79776 & 483.4128418 & 275.4145508 & 272.3617249 & 131.6789246 \\
Killarney Park & 3160.15918 & 8609.833008 & 1402.926758 & 1428.557617 & 7316.314453 & 1892.266602 \\
McFetridge Park & 5461.837891 & 103.5576172 & 4819.770508 & 36.79882813 & 1404.405273 & 1979.706055 \\
Normanton Reserve & 544.5351563 & 1909.788086 & 354.5947266 & 373.9892578 & 1688.322266 & 342.4638672 \\
Puhinui Domain & 19511.99994 & 69455.7518 & 56116.37057 & 82893.59921 & 2093.553741 & 28110.97137 \\
Robert White Park & 0 & 0 & 0 & 0 & 0 & 0 \\
Russell Road Reserve & 1511.267151 & 9524.483978 & 1052.96701 & 7910.964752 & 1634.870209 & 479.6509399 \\
Stancich Reserve & 824200.4697 & 1560491.801 & 64279.37207 & 2873.81543 & 819018.793 & 50225.59277 \\
Tadmoor Park & 8388.599365 & 3037.15976 & 3787.05957 & 733.4524231 & 11932.83618 & 1146.519043 \\
Taharoto Park & 743.9765625 & 37836.19238 & 321.2314453 & 37842.42578 & 323.984375 & 38055.57227 \\
Teviot Reserve & 297.4169922 & 69.4296875 & 406.0068359 & 78.33398438 & 263.484375 & 163.7978516 \\
\hline
\end{tabular}

The results of the pixel-based experiments show the differences between two views (sets of boundary data) for the same area. The first set of differences shows the encroachment of private land onto public land and the second set of differences shows the encroachment of public land onto private land. This approach highlights the differences between the boundaries but does not propose any possible solutions to the problem. The results of the polygon-based experiments in Table 1 show the plotting of the two sets of points and highlights the differences between the two views (sets of boundary data) for the same area. AreaOmG gives the area of the original boundary minus that of the GPS boundary. AreaGmO gives the area of the GPS boundary minus that of the original boundary. AreaOmM gives the area of the original boundary minus that of the middle boundary. AreaMmO gives the area of the middle boundary minus that of the original boundary. AreaGmM gives the area of the GPS boundary minus that of the Middle boundary. AreaMmG gives the area of the middle boundary minus that of the GPS boundary. The results of the experiments show the differences between two views (sets of boundary data) for the same area. We give a fair solution to people who have arguments on the measurement of boundary for Parks in Auckland, as seen in Fig. 3. The green line shows the boundary as perceived by the council and the blue line shows the boundary as perceived by the results of a field survey. The differences in the two boundaries show where the boundary is disputed. The red line shows a calculated middle boundary which may act as a starting point for resolving boundary disputes. Stakeholders have different views on encroachment. For example the council have a strict viewpoint and assume that the data they have is correct whereas some private residents have a relaxed viewpoint on the position of a boundary. 


\section{Conclusions and Future Work}

In order to address the land encroachment problems in Auckland's parks, we firstly proposed two different approaches boundary calculations. Though both of them detect and highlight differences, in numerical terms, effectively, whereas neither approach offers a possbile solution to any boundary conflicts. We then proposed the middle-boundary approach, in which we address the boundary arguments from stakeholders by a nearest neighbor based algorithm. This solution could be a possible solution that disputes or at least a starting point for negotiations, because the (middle-boundary) have been addressed. The main limitation of the middle-boundary approach is that the proposed new boundary is based solely on mathematical calculations and does not take into account the stakeholders' views or motivations currently. Possible future work could involve adding weighting to the middle-boundary calculations so that different possible solutions could be generated.

\section{References}

1. D. F. Shmueli, "Framing in geographical analysis of environmental conflicts: Theory, methodology and three case studies," Geoforum, vol. 39, no. 6, pp. 2048-2061, 2008.

2. Y. He and A. Kundu, "2-d shape classification using hidden markov model," IEEE Transactions on Pattern Analysis and Machine Intelligence, vol. 13, no. 11, pp. 1172-1184, 1991.

3. M. Bicego and V. Murino, "Investigating hidden markov models' capabilities in 2-d shape classification," IEEE Transactions on Pattern Analysis and Machine Intelligence, vol. 26, no. 2, pp. 281-287, 2004.

4. I. Deneme, H. Yerli, M. Severcan, A. Tanrikulu, and A. Tanrikulu, "Use and comparison of different types of boundary elements for $2 \mathrm{~d}$ soil-structure interaction problems," Advances in Engineering Software, vol. 40, pp. 847-855, 2009.

5. H. Rom and G. Medioni, "Hierarchical decomposition and axial shape description," IEEE Transactions on Pattern Analysis and Machine Intelligence, vol. 15, no. 10, pp. 973-981, 1993.

6. Y. Liu, Y. Yuan, D. Xiao, Y. Zhang, and J. Hu, "A point-set-based approximation for areal objects: A case study of representing localities," Computers, Environment and Urban Systems, vol. 34, pp. 28-39, 2010.

7. S. Stehman, "Estimating area from an accuracy assessment error matrix," Remote Sensing of Environment, vol. 132, pp. 202-211, 2013.

8. F. Baffetta, L. Fattorini, S. Franceschi, and P. Corona, "Design-based approach to k-nearest neighbours technique for coupling field and remotely sensed data in forest surveys," Remote Sensing of Environment, vol. 113, pp. 463-475, 2009.

9. A. von der Dunk, A. Grêt-Regamey, T. Dalang, and A. M. Hersperger, "Defining a typology of peri-urban land-use conflicts-a case study from switzerland," Landscape and Urban Planning, vol. 101, no. 2, pp. 149-156, 2011.

10. B. Forest, "Information sovereignty and gis: the evolution of communities of interest in political redistricting," Political Geography, vol. 23, no. 4, pp. 425-451, 2004. 